\title{
Indirect Interactions between Raman Probes Encapsulated within Cucurbit[7]urils and Gold Nanorods to Enhance Long-term Stability and Signal
}

\author{
Min Jung SEO,* Kangkyun BAEK, ${ }^{* *}$ and Ji Won HA ${ }^{* \dagger}$ \\ *Advanced Nano Bio Imaging and Spectroscopy Laboratory, Department of Chemistry, University of Ulsan, \\ 93 Daehak-Ro, Nam-Gu, Ulsan 44610, Republic of Korea \\ **Center for Self-assembly and Complexity, Institute for Basic Science, Pohang 37673, Republic of Korea
}

\begin{abstract}
Surface-enhanced Raman scattering (SERS) is a powerful technique that enhances Raman signals by adsorbing probe molecules on rough metal surfaces. However, SERS is limited because target molecules must strongly interact with metal to enhance a stable Raman signal. In this study, to improve long-term SERS stability, we use cucurbit[7]urils (CB[7]) as bridge molecules and sample containers to probe Rhodamine 6G (R6G) molecules. We observed interactions between gold nanorods (AuNRs) and CB[7] via aggregate formation, which enhanced the Raman signal and improved long-term R6G probe stability by up to 20 days when encapsulated within CB[7] during SERS analysis.
\end{abstract}

Keywords Surface-enhanced Raman scattering, cucurbit[7]urils, Rhodamine 6G, long-term stability

(Received April 24, 2019; Accepted May 14, 2019; Advance Publication Released Online by J-STAGE May 24, 2019)

\section{Introduction}

Raman spectroscopy, combined with infrared spectroscopy, is a powerful spectroscopic technique that provides information on vibrational modes. ${ }^{1}$ However, Raman spectroscopy is disadvantageous because spontaneous Raman scattering is typically very weak. ${ }^{2-4}$ Surface-enhanced Raman scattering (SERS) is one method that can overcome the disadvantages of traditional Raman spectroscopy. ${ }^{2-6}$ SERS is a technique that powerfully enhances Raman signals by adsorbing target molecules on rough metal surfaces, which are most commonly metal nanoparticles. ${ }^{5,7-9}$ However, SERS is also limited because of strong interactions between the Raman probe molecules and the metal surface, which are necessary for the stable enhancement of the signal. To improve the long-term stability of Raman enhancements, previous studies have attempted to develop additional approaches and strategies, such as the introduction of a new host-guest chemistry and hybridization systems. ${ }^{10-13}$

Cucurbit $[n]$ urils $(\mathrm{CB}[n])$ are macrocyclic host molecules composed of glycoluril repeat units bridged by methylene groups (Fig. 1). Compared with other host molecules, such as cyclodextrin and crown ethers, $\mathrm{CB}[n]$ molecules have unique properties that are contained within rigid, highly symmetric, and macrocyclic pumpkin-shaped structures. ${ }^{14-16}$ Due to the hollow and cyclic structure of $\mathrm{CB}[n]$, target molecules position themselves inside the hydrophobic cavity of $\mathrm{CB}[n]$ via strong host-guest interactions. ${ }^{15,16}$ Furthermore, $\mathrm{CB}[n]$ can attach itself onto gold surfaces through the interaction between the carbonyl groups of $\mathrm{CB}[n]$ and $\mathrm{Au}$ surface, which leads to the formation of

† To whom correspondence should be addressed.

E-mail: jwha77@ulsan.ac.kr uniform and stable sub-nanometer gaps at $0.9 \mathrm{~nm}$, i.e., the height of the $\mathrm{CB}[n] .{ }^{17}$ This indicates that $\mathrm{CB}[n]$ induces a powerful hotspot between nanoparticles that is highly effective for SERS. ${ }^{18}$ The encapsulation inside $\mathrm{CB}[n]$ may also contribute to the improved stability of the SERS structure and provide possible enhancements without direct interaction between metal nanoparticles and probe molecules. The carbonyl portals of $\mathrm{CB}[n]$, placed on the metal surface, allow indirect interactions due to a trapped state related to electromagnetic enhancements, which is also related to the SERS mechanism. ${ }^{19}$

Despite recent studies on SERS that use $\mathrm{CB}[n]$, the interactions between $\mathrm{CB}[n]$ and various guest molecules to initiate effective SERS and the enhancement mechanisms behind SERS based on $\mathrm{CB}[n]$ and gold nanoparticles still lack fundamental understanding. Furthermore, the long-term stability and reproducibility of SERS signals for various Raman probes (e.g., R6G), which are encapsulated within $\mathrm{CB}[n]$ that is bound to gold nanoparticle surfaces, still require investigation to improve

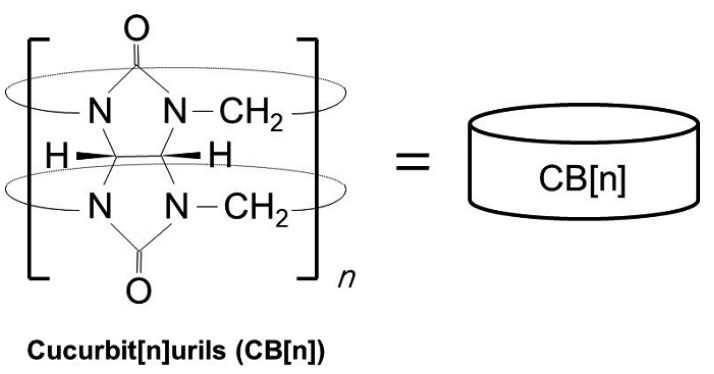

Fig. 1 A schematic displaying the repeating units and a representation of $\mathrm{CB}[n]$. 
SERS stability in several biological applications.

Herein, we introduced $\mathrm{CB}$ [7] to overcome SERS limitations, such as unspecific adsorption, weak interactions, or low stability with gold surfaces, which all lead to difficulties when using SERS. ${ }^{20}$ We sought to produce stable AuNR aggregations using $\mathrm{CB}[7]$ to yield regular alignments to understand the role of $\mathrm{CB}$ [7] and its interaction with AuNRs. Moreover, we performed a SERS study of R6G with the AuNR aggregates; CB[7] acts as a container to discern the long-term stability of this system. $\mathrm{CB}$ [7] allowed us to stably capture R6G in the $\mathrm{CB}$ [7] cavity, which we verified with stable SERS signals.

\section{Experimental}

\section{Chemicals and materials}

Gold nanorods (AuNRs), with an average size of $25 \times 73 \mathrm{~nm}$, were purchased from Nanopartz (Loveland, CO, USA). Rhodamine 6G (R6G), used as a Raman probe, was obtained from Sigma-Aldrich (St. Louis, MO, USA). Cucurbit[7]uril $(\mathrm{CB}[7])$ was synthesized by following the procedure as described previously. $^{21}$

\section{AuNR characterization}

The shape and size of the AuNRs were characterized using a scanning electron microscope (SEM) (JEOL, Tokyo, Japan) at $10 \mathrm{kV}$. UV-Vis extinction spectra for the AuNRs were collected in a quartz absorption cell with a Varian Cary $300 \mathrm{UV}-\mathrm{Vis}$ spectrophotometer (Agilent, CA, USA). For UV-Vis measurements, the AuNR solution obtained from the Nanopartz was used without dilution.

\section{Sample peparation for Raman measurements}

For bulk samples in vials, $400 \mu \mathrm{L}$ of an aqueous $1 \mathrm{mM} \mathrm{CB}$ [7] solution was added to a $4 \mathrm{~mL}$ AuNR solution. A pure AuNR solution (without $\mathrm{CB}[n]$ ) was used as a control. One of the two SERS samples was prepared by mixing a $100-\mu \mathrm{L}$ AuNR solution with $100 \mu \mathrm{L}$ of the $1 \mathrm{mM}$ R6G solution. The second sample was prepared via the addition of a $100-\mu \mathrm{L}$ AuNR solution to the solution of R6G encapsulated within the CB[7] cavity. The volume and concentrations of the $\mathrm{CB}[7]$ and $\mathrm{R} 6 \mathrm{G}$ solutions in the two different samples were kept constant for comparison. R6G solutions were made with $18.2 \mathrm{M} \Omega$ pure water at the proper concentration for each measurement. In the encapsulation sample, the molar ratio between $\mathrm{CB}[7]$ and $\mathrm{R} 6 \mathrm{G}$ was $1: 1$ because $\mathrm{CB}$ [7] can only contain one molecule inside the cavity. The prepared samples were placed into capillary tubes with a diameter of $0.25 \mathrm{~mm}$ and a length of $100 \mathrm{~mm}$. These two samples were utilized for SERS measurement after being kept at room temperature for $6 \mathrm{~h}, 1$ day, 8 days, and 20 days.

\section{Raman instrumentation}

We used a custom-made Raman spectroscopic system, equipped with a 785-nm diode laser (Thorlabs, Newton, NJ, USA) to produce the excitation light, for SERS measurements (Figs. S1 and S2, Supporting Information). A capillary tube was used as a sample container during the SERS measurements (Fig. S3, SI). The capillary tubes containing the samples were placed onto the microscope stage, and the laser power was set to $\sim 60 \mathrm{~mW}$. However, the actual amount of power that arrived at the sample was approximately half of the initial power. The Raman spectra were collected with an Andor spectrometer (SHAMROCK 303i, UK) connected to an Andor CCD camera (Newton Du9209-OE, UK). Raman sample signals were collected using a $40 \times$ objective lens with a 0.75 numerical

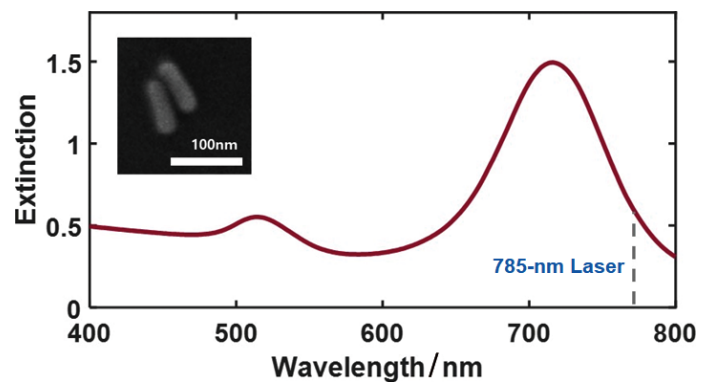

Fig. 2 The UV-Vis extinction spectrum for AuNRs with an average size of $25 \times 73 \mathrm{~nm}$. The dotted line indicates the $785 \mathrm{~nm}$ laser wavelength used as the resonance condition for this SERS study. The inset shows an enlarged SEM image of single AuNRs.

aperture (NA). The monochromator was used in combination with $600 \mathrm{l} / \mathrm{mm}$ grating while the slit width was approximately $100 \mu \mathrm{m}$.

\section{Results and Discussion}

In this study, plasmonic AuNRs were used as SERS substrates for the following reasons: 1) AuNRs are photostable and biocompatible, and we can easily control their unique optical properties by tuning their aspect ratios; 2) AuNRs are beneficial during SERS analysis due to strong lighting effects that may result in higher local field enhancement as well as SERS signal enhancement; 3) the AuNR surface is easily modified with molecules and other metals via previously established wet chemistry techniques; and 4) an AuNR has three different types of facets that are connected with surface energies and, therefore, favors adsorption to molecules on the surface, which then forms stable complexes.

We first characterized the shapes and sizes of the AuNRs with SEM analyses. As shown in the inset of Fig. 2, anisotropic AuNRs obtained from Nanopartz had an average size of $25 \times$ $73 \mathrm{~nm}$. We then measured the UV-Vis extinction spectrum of the AuNRs dispersed in water with a Varian Cary $300 \mathrm{UV}$-Vis spectrophotometer, also shown in Fig. 2. The spectrum has two characteristic LSPR peaks at approximately 520 and $711 \mathrm{~nm}$. The left peak (i.e., high energy or low wavelength) occurs due to conduction electrons that exhibit transverse oscillation, whereas the right peak (i.e., low energy or high wavelength) results from longitudinal LSPR oscillation.

As shown in Fig. 2, the broad longitudinal LSPR peak at approximately $711 \mathrm{~nm}$ occurs due to heterogeneity in the nanoparticle sizes. More importantly, the $785 \mathrm{~nm}$ radiation is near the tail of the longitudinal peak, which indicates that we do not expect the presence of maximized resonance effects in this SERS study. However, a 785-nm laser wavelength is located inside the longitudinal LSPR peak, which indicates that a resonance effect may still occur between the AuNRs and the Raman laser. This observation also suggests that electromagnetically enhanced signals are possible for the AuNRs at $785 \mathrm{~nm}$.

One method to enhance the Raman signal is the use of hotspots that may form between gold nanoparticles or between the gold nanoparticle and gold film. ${ }^{17,20,22}$ In this study, we used CB[7] as a bridge molecule to form aggregations between anisotropic AuNRs and hotspots to improve SERS. Here, we used CB[7] for the following two reasons: 1) the rigid and symmetric $\mathrm{CB}$ [7] 
A

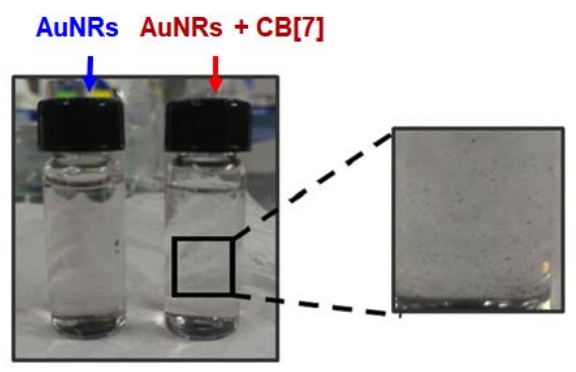

B
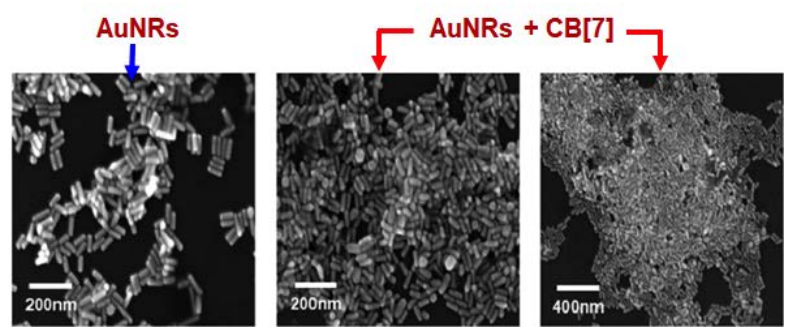

Fig. 3 (A) A bulk AuNR sample (left) and an AuNR sample that contains CB[7]. (B) SEM images of AuNRs without CB[7] (left) and AuNRs with $\mathrm{CB}$ [7] (the two images on the right).

structure and its carbonyl portals can efficiently interact with the gold surface by replacing the capping ligand coated on gold through the interaction between carbonyl and gold, ${ }^{23}$ which may yield uniform and stable sub-nanometer gaps of $0.9 \mathrm{~nm}$ that previous studies have suggested to be the best gap distance for SERS hotspots, ${ }^{23-25}$ and 2) the hollow cyclic structure of $\mathrm{CB}$ [7] has the ability to form ultra-stable complexes with multiple guests. ${ }^{26}$ The strong hydrophobic interaction between the host cavity and guests, and the ion-dipole and dipole-dipole interactions between the guests and CB portals promote the cooperative and multiple noncovalent interactions that are essential to produce such strong complexations. Therefore, strong encapsulation can contribute to the enhanced stability of the Raman probe molecules (or guest molecules) inside CB[7] during SERS.

As depicted in Fig. S4 (SI), we obtained a number of results on CB[7]'s role as a bridge molecule in forming AuNR aggregates. A $400-\mu \mathrm{L}$ aqueous solution of $1 \mathrm{mM} \mathrm{CB}$ [7] was added to a 4-mL AuNR solution, and a pure AuNR solution (without $\mathrm{CB}[\mathrm{n}]$ ) with an identical concentration was used as the control. Figure 3A shows two vials containing the AuNR solution with and without the $\mathrm{CB}$ [7]. We observed, with the naked eye, that the AuNR solution without $\mathrm{CB}$ [7] had a reddish color and was clear without aggregations. In contrast, the AuNR solution containing $\mathrm{CB}[7]$ was completely colorless with several floating particles. We also observed aggregations as indicated in the enlarged picture in Fig. S4 (SI). For a detailed examination of the two different samples, we used a SEM analysis, as shown in Fig. 3B. At an identical SEM magnification, the sample with $\mathrm{CB}$ [7] contained an elevated amount of aggregated nanoparticles compared with the sample without $\mathrm{CB}$ [7], which contained only AuNRs. In addition, the SEM image at a smaller magnification (or wider view) was characterized by a higher tendency to form aggregations. More importantly, we observed the elevated aggregation tendency, in the presence of $\mathrm{CB}$ [7], for a number of other regions in the same sample during SEM imaging analysis.

To further understand the formation mechanism of the AuNR aggregates, we performed UV-Vis spectroscopy measurements. We obtained UV-Vis extinction spectra from three types of
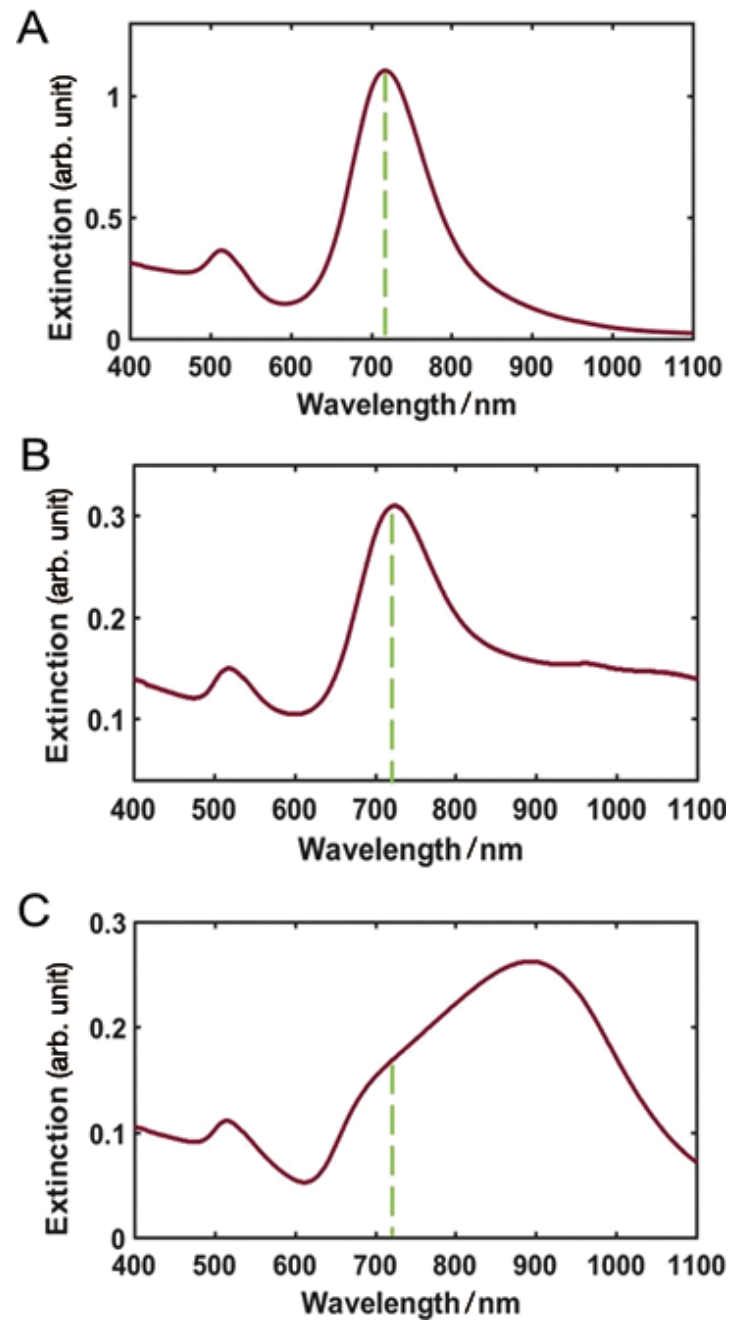

Fig. 4 The UV-Vis extinction spectra of (A) raw AuNRs, (B) AuNRs after centrifugation, and (C) AuNRs with $\mathrm{CB}$ [7].

samples: 1) pure AuNRs without CB[7], 2) centrifuged AuNRs without $\mathrm{CB}$ [7], and 3) AuNRs with $\mathrm{CB}$ [7]. We then compared the UV-Vis spectra for each of the three different samples, as shown in Fig. 4. Here, we used the centrifuged AuNR sample without $\mathrm{CB}[7]$ to confirm that this step may cause LSPR peak broadening due to AuNR aggregations, which are a consequence of surfactant removal that protects particle surfaces. When we compared the pure (Fig. 4A) and centrifuged AuNRs (Fig. 4B), we observed the broadening of the longitudinal peak in the centrifuged AuNRs, which indicates the formation of aggregations. However, the position of the longitudinal LSPR peak for the centrifuged sample was nearly identical to the position of the pure AuNR sample without any significant frequency shift. These results indicate that centrifugation causes no significant effects on the spectra although it may cause certain AuNR aggregations. For the AuNRs with CB[7], however, the longitudinal LSPR peak was significantly redshifted (Fig. 4C). Furthermore, the longitudinal LSPR peak was characterized by a large broadening with a small shoulder peak at approximately $700 \mathrm{~nm}$ at the pure AuNR longitudinal LSPR wavelength, as indicated in Fig. 4A. Besides the longitudinal LSPR peak, the transverse LSPR peak, at approximately $517 \mathrm{~nm}$ in Fig. 4C (AuNRs and CB[7]), had a relatively lower peak height due to a decrease in intensity during transverse mode with the formation of aggregations. Therefore, 

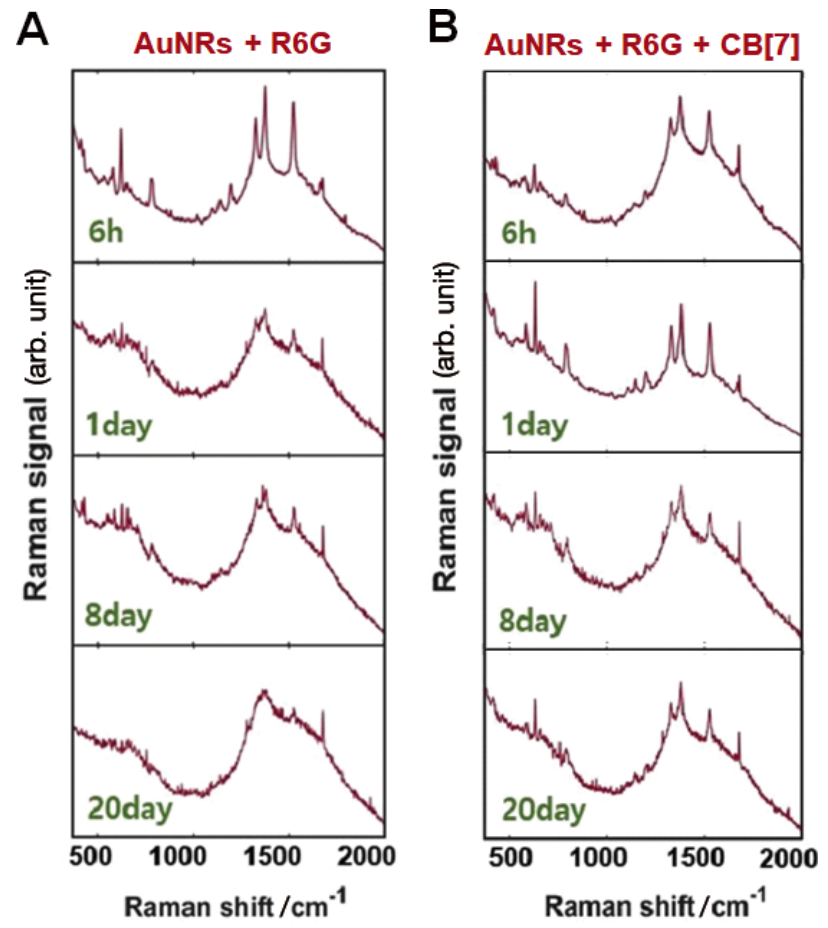

Fig. 5 (A) SERS spectra of the AuNRs and R6G without CB[7] measured repeatedly after $6 \mathrm{~h}, 1$ day, 8 days, and 20 days. (B) SERS spectra of the AuNRs and R6G encapsulated within the CB[7] and measured after $6 \mathrm{~h}, 1$ day, 8 days, and 20 days with exposure to identical experimental conditions.

the UV-Vis spectroscopic data supports the fact that $\mathrm{CB}$ [7] can efficiently bind to AuNR surfaces and that AuNRs can easily form aggregations in the presence of $\mathrm{CB}$ [7] bridge molecules.

In our previous study, R6G, a dye molecule, efficiently bound to $\mathrm{Au}$ nanourchin branched surfaces via its positive nitrogen. ${ }^{9}$ However, after a binding time of $6 \mathrm{~h}$, the resolution was poor due to weak interactions between the metals and probe molecules. ${ }^{9}$ Therefore, we suggest that R6G molecules can interact with $\mathrm{Au}$ nanoparticles that have low stability. This previous study motivated our search to improve the stability of R6G molecules that are attached to Au nanoparticles to improve SERS

To improve the low stability of R6G on metal surfaces, we used $\mathrm{CB}$ [7] molecules as a container. The host-guest interaction between $\mathrm{CB}$ [7] and R6G can cause the encapsulation of R6G inside a $\mathrm{CB}$ [7] cavity. ${ }^{9,15,26-28}$ We thus investigated $\mathrm{CB}$ [7] performance and properties as a container with the aim of maintaining an enhanced SERS signal, as well as obtaining long-term R6G stability on metal surfaces. We prepared two samples and compared them to understand the effects that encapsulation has on the stability of R6G during SERS measurements (Fig. S5, SI). Figure S5A (SI) shows the direct interaction between R6G and the AuNR surfaces, whereas Fig. S5B (SI) depicts the indirect interaction between R6G and the AuNRs via encapsulation using $\mathrm{CB}[7]$. The prepared samples were placed into capillary tubes that were $0.25 \mathrm{~mm}$ in diameter and $100 \mathrm{~mm}$ in length (Fig. S3, SI) and were measured at room temperature after $6 \mathrm{~h}, 1$ day, 8 days, and 20 days while exposed to identical experimental conditions. Figure 5 shows the corresponding SERS spectra. In the spectra recorded after $6 \mathrm{~h}$, we observed distinct Raman peaks for the two samples despite interference from the capillary tubes, which indicates efficient R6G adsorption, as well as SERS enhancement. Note

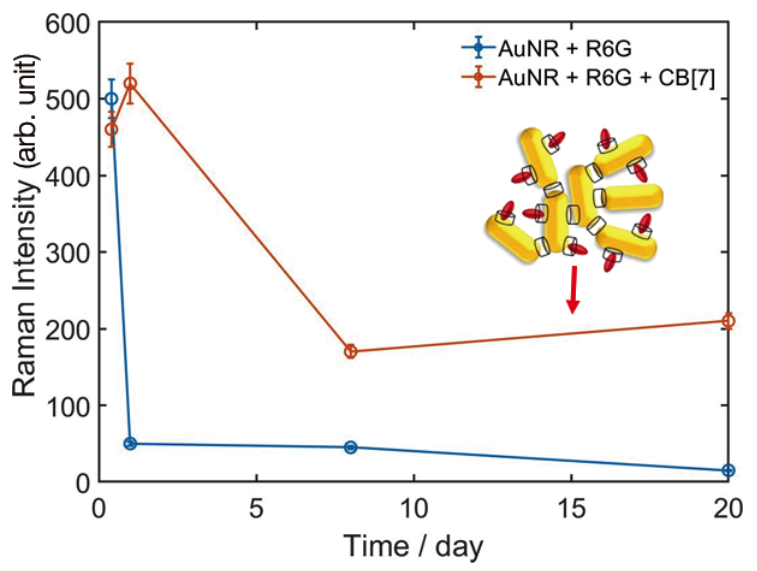

Fig. 6 Changes in the Raman intensity of AuNRs and R6G (blue line) and AuNRs with R6G encapsulated within CB[7] (red line) that were measured repeatedly after 6 h, 1 day, 8 days, and 20 days. A peak at $1507 \mathrm{~cm}^{-1}$ was used to compare the changes in the Raman intensity.

that the broad bands between 1200 and $1600 \mathrm{~cm}^{-1}$ are a consequence of the capillary tubes and are not the R6G probe Raman signals. However, in the sample without CB[7], Raman signals had decreased significantly after 1 day, and the distinctive R6G peaks nearly disappeared after 20 days (Figs. 5A and 6). These results display the low degree of long-term stability for R6G that is attached to the AuNR surfaces. In contrast, the sample with $\mathrm{CB}[7]$ was characterized by distinctly high Raman peaks in the spectrum even after 20 days (Figs. 5B and 6). These results suggest that $\mathrm{CB}[7]$ is an efficient container during SERS although the probe molecules did not interact with the metal surfaces directly. In other words, indirect interactions between the Raman probes and metal surfaces, in the presence of $\mathrm{CB}$ [7], enhance the Raman signal and long-term stability.

\section{Conclusions}

In conclusion, this study attempted to introduce $\mathrm{CB}$ [7] with cavities as a solution to control SERS limitations associated with unspecific Raman molecules, such as weak interactions or low stability along gold surfaces, which yield difficulties during SERS analysis. We investigated the formation of AuNR aggregations in the presence of $\mathrm{CB}$ [7] acting as bridge molecules. Furthermore, we observed that $\mathrm{CB}$ [7] has significant potential as a sample container. Their use results in long-term R6G stability for up to 20 days and the enhancement of SERS measurements. Therefore, we demonstrated that these molecules, which have faced numerous complications during SERS applications due to their insolubility or inability to bind to a metal surface, are now usable during SERS in combination with the CB[7] containers. Indirect interactions between Raman probes and metal surfaces may lead to efficient signal enhancement and improvements in long-term stability.

\section{Acknowledgements}

J. W. $\mathrm{H}$ is grateful for the National Research Foundation of Korea (NRF) grant funded by the Korean government (MSIP) (No. 2018R1C1B3001154). We thank Prof. Kimoon Kim at Pohang University of Science and Technology for donating $\mathrm{CB}[7]$ and helpful discussions. 


\section{Supporting Information}

This document contains supporting figures (Figs. S1 to S5). This material is available free of charge on the Web at http:// www.jsac.or.jp/analsci/.

\section{References}

1. P. J. Hendra and P. M. Stratton, Chem. Rev., 1969, 69, 325.

2. S. He, J. Chua, E. K. M. Tan, and J. C. Y. Kah, $R S C A d v$, 2017, 7, 16264.

3. P. Hildebrandt and M. Stockburger, J. Phys. Chem., 1984, 88, 5935 .

4. S. Pahlow, T. Mayerhöfer, M. van der Loh, U. Hübner, J. Dellith, K. Weber, and J. Popp, Anal. Chem., 2018, 90, 9025.

5. K. Kneipp, Y. Wang, H. Kneipp, L. T. Perelman, I. Itzkan, R. R. Dasari, and M. S. Feld, Phys. Rev. Lett., 1997, 78, 1667.

6. M. Suzuki, Y. Niidome, N. Terasaki, K. Inoue, Y. Kuwahara, and S. Yamada, Jpn. J. Appl. Phys., 2004, 43, L554.

7. G. Macias, M. Alba, L. F. Marsal, and A. Mihi, J. Mater. Chem. C, 2016, 4, 3970.

8. G. Xue, J. Dong, and J. Zhang, Macromolecules, 1992, 25, 5855.

9. M. Seo and J. W. Ha, Microchem. J., 2018, 140, 47.

10. A. Feofanov, A. Ianoul, E. Kryukov, S. Maskevich, G. Vasiliuk, L. Kivach, and I. Nabiev, Anal. Chem., 1997, 69, 3731.

11. M. Nowak, A. Trojanowska, L. Marciniak, M. Binczyk, T. Runka, B. Tylkowski, and R. Jastrzab, Appl. Surf. Sci., 2018, 472, 93.

12. Y. Liu, H. Wu, L. Ma, S. Zou, Y. Ling, and Z. Zhang, Nanoscale, 2018, 10, 19863.
13. M. Zhao, H. Guo, W. Liu, J. Tang, L. Wang, B. Zhang, C. Xue, J. Liu, and W. Zhang, Nanoscale Res. Lett., 2016, 11, 403.

14. K. M. Park, K. Baek, Y. H. Ko, A. Shrinidhi, J. Murray, W. H. Jang, K. H. Kim, J.-S. Lee, J. Yoo, S. Kim, and K. Kim, Angew. Chem. Int. Ed., 2018, 57, 3132.

15. S. J. Barrow, S. Kasera, M. J. Rowland, J. del Barrio, and O. A. Scherman, Chem. Rev., 2015, 115, 12320.

16. E. Masson, X. Ling, R. Joseph, L. Kyeremeh-Mensah, and $\mathrm{X} . \mathrm{Lu}, \mathrm{RSC} A d v$., 2012, 2, 1213.

17. N. H. Kim, W. Hwang, K. Baek, M. R. Rohman, J. Kim, H. W. Kim, J. Mun, S. Y. Lee, G. Yun, and J. Murray, J. Am. Chem. Soc., 2018, 140, 4705.

18. T.-C. Lee and O. A. Scherman, Chem. Commun., 2010, 46 , 2438.

19. S. T. Jones, R. W. Taylor, R. Esteban, E. K. Abo-Hamed, P. H. Bomans, N. A. Sommerdijk, J. Aizpurua, J. J. Baumberg, and O. A. Scherman, Small, 2014, 10, 4298.

20. C.-a. Tao, Q. An, W. Zhu, H. Yang, W. Li, C. Lin, D. Xu, and G. Li, Chem. Commun., 2011, 47, 9867.

21. J. Kim, I.-S. Jung, S.-Y. Kim, E. Lee, J.-K. Kang, S. Sakamoto, K. Yamaguchi, and K. Kim, J. Am. Chem. Soc., 2000, 122, 540 .

22. D. Radziuka and H. Moehwald, Nanoscale, 2014, 6, 6115.

23. Q. An, G. Li, C. Tao, Y. Li, Y. Wu, and W. Zhang, Chem. Commun., 2008, 17, 1989.

24. S. Mahajan, T.-C. Lee, F. Biedermann, J. T. Hugall, J. J. Baumberg, and O. A. Scherman, Phys. Chem. Chem. Phys., 2010, 12, 10429.

25. G. Y. Tonga, T. Mizuhara, K. Saha, Z. Jiang, S. Hou, R. Das, and V. M. Rotello, Tetrahedron Lett., 2015, 56, 3653.

26. D. Shetty, J. K. Khedkar, K. M. Park, and K. Kim, Chem. Soc. Rev., 2015, 44, 8747.

27. J. Mohanty and W. M. Nau, Angew. Chem. Int. Ed., 2005, 44,3750 .

28. A. L. Koner, and W. M. Nau, Supramol. Chem., 2007, 19, 55. 